\title{
STOCHASTIC MODELLING FOR RISK ASSESSMENT
}

\author{
YAPING HE, MAHMUT HORASAN, PETER TAYLOR and CAIRD RAMSAY \\ Scientific Services Laboratory \\ 177 Salmon Street, Port Melbourne, VIC 3207 \\ Australia
}

\begin{abstract}
A probabilistic risk assessment method is described in this paper for the analysis of expected risk to life. The method involves stochastic, as well as deterministic, modelling of building occupant evacuation process and the attainment of untenable conditions due to fires in buildings. Both the number of occupants leaving a building at a given time and the time to untenable conditions are treated as random variables. A general expression for calculating the expected number of death is given. With the postulated Poisson distribution for the number of occupants leaving a building and uniform distribution for the time to untenability, a simple expression for the calculation of the expected number of deaths is obtained. An example is given to illustrate the application of the method.
\end{abstract}

KEY WORDS: uncertainty, expected risk to life, event tree, probabilistic approach

\section{INTRODUCTION}

Fire safety engineering has made a great deal of progress in the past two decade along with the transition from prescriptive building codes to performance-based building codes in many parts of the world. It has evolved from a discipline with limited science and largely empirical basis to a largely scientific based multi-disciplinary engineering. Its maturity is measured by the growing sophistication in the quantitative as well as qualitative methodologies adopted in the design and evaluation processes to cope with the demands of building design and the construction industry.

Fire engineering methodologies for evaluating the performance and hence assisting in the design of the safety systems can be generally classified into two categories, namely, the deterministic and the probabilistic. Fire safety engineering in essence is risk reduction engineering. The objective of fire safety engineering design is to minimise the risk to life and/or to property. The task of quantifying risk is then a pre-requisite for risk reduction.

The risk concept has been accepted by engineers, scientists and the like in dealing with safety. For example, even with the traditional deterministic system performance evaluation method, an engineer is often inclined to use safety factors or safety margins to account for uncertainties [1]. The traditional deterministic approach alone is, however, not capable of giving quantified risk assessment without involving a high degree of empiricism The fact that any type of fire safety engineering assessment will inevitably involve probabilistic approach to various degrees has been recognised in the latest edition of the Australian Fire Safety Engineering Guidelines [1].

A risk-based methodology provides a systematic approach for quantifying risks involving complex systems or phenomena [2]. The probabilistic concept and the method of 
stochastic analysis have been used in engineering designs for traffic, dam and many other engineering fields [3]. Magnusson [4] presented a general summary of the probabilistic methods for risk assessment in the field of fire safety engineering. Applications of these methods for assessing and comparing the adequacy of essentially different fire protection strategies (e.g., sprinklers and compartmentation) can also be found in the literature [5].

Risk is defined as the product of the frequency of event and the expected consequences [6]. Risk assessment is accordingly divided into two main parts: determination of frequency of initiating event and determination of consequences of initiating event occurring [4]. The former involves research and analysis of statistics. The latter relies on the studies of the methods for evaluating the consequences.

One of the documented risk assessment methods is referred as the reliability index method [5, 7]. This method, when applied to the single scenario case in fire safety engineering, treats the safety margin, which is the difference between the available safe egress time (ASET) and the required safe egress time (RSET), as a random variable and the ratio of its mean to its standard deviation is seen as the reliability index. The probability of failure can be calculated as a function of the reliability index. This method was expanded by Hasofer and Beck [8] to estimate the death in the room of fire origin. In their formulation of the method, the expected number of deaths, $D$, was calculated by

$$
D=L p,
$$

where $L$ is the number of occupants in the room of fire origin and $p$ is the probability that the safety margin is less than zero. The above expression is a good approximation if the number of the occupants in the room is small, or the occupants evacuate the room in a more or less simultaneous fashion. If there are a significant number of occupants and the evacuation process is extended for a time period, the number of occupants remaining in an enclosure, $N$, is itself a function of time and a random variable. For example, during a fire incident in a large office building, the entire floor of fire origin may become untenable in a given time range. The evacuation process will have a time span and the number of the occupants remaining on the floor decreases with time. The number of fatalities not only depends on whether the safety margin is negative but also on by how much the ASET is smaller than the RSET. In this case, a more appropriate formulation for estimating the expected number of deaths or fatalities is needed.

In the current study, a method incorporating stochastic modelling technique for calculating the expected number of deaths and risk assessment is discussed. Both the occupant evacuation and the attainment of untenable conditions in buildings subjected to fires are treated as random processes. Instead of uncertainty analysis of the required safe egress time, this method focus on the analysis of the number of occupants remaining in the building at any given time as random variable. Stochastic models are established with the prescribed probability distribution function for egress, or more specifically, occupant movement process and probability density distribution function for time to untenability.

\section{TIME-LINE APPROACH AND TREATMENT OF UNCERTAINTY}

Fatalities in a fire are results of certain events occurring in a particular sequence. In a fire scenario, if RSET is greater than ASET, then fatalities are deemed to occur. This time line approach is illustrated in Figure 1, where $t_{\mathrm{d}}$ is time of fire detection, $t_{\mathrm{p}}$ is premovement time interval, $t_{\mathrm{e}}$ is time of commencement of egress, $t_{\mathrm{t}}$ is occupant travel time 
interval, $t_{\mathrm{r}}$ is RSET and $t_{\mathrm{u}}$ is time of onset of untenable conditions.

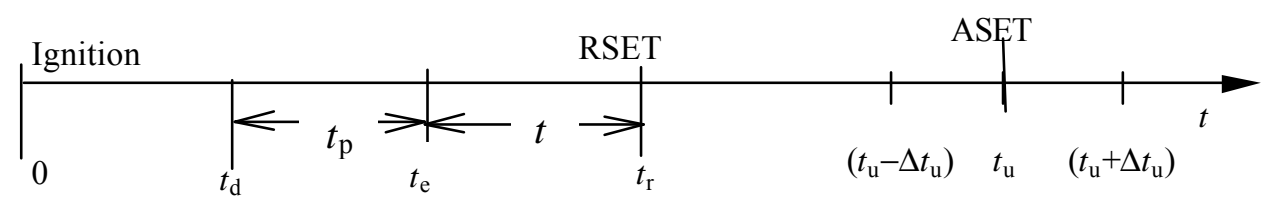

Figure 1. Time-line

The following relation between various time components holds

$$
t_{r}=t_{e}+t_{t}=t_{d}+t_{p}+t_{t} .
$$

The safety margin $S$ is defined as

$$
S=t_{u}-t_{r}
$$

In the time-line approach, the acceptance criterion for a design is

$$
S>0, \text { or } t_{r}<t_{u}
$$

The required safe egress time $t_{r}$ corresponds to the time of the last occupant leaving a designated enclosure or a building. If the above criterion is satisfied, then a nil fatality is expected. This approach makes sense only within the deterministic framework. In reality, both $t_{r}$ and $t_{u}$ are random variables. In general, the times of events in a fire may not be certain. There will be a range of times over which an event may occur. For example, the failure time of a smoke barrier with nominated fire rating may vary even for a nominated fire. The uncertainty in the barrier failure time will result in the uncertainty in the time to untenable conditions in the enclosures of interest. The degree of this latter uncertainty will increase if the fire safety system in a building involves multiple components. Generally, the greater is the complexity of a system, the greater the uncertainty in its performance.

Human behaviour in fire emergencies is by far the most complex phenomenon for fire safety engineering analysis. Computerised models dealing with such phenomenon have to introduce simplifying assumptions in order to derive results with available means. These assumptions are related to the analytical methods and have their basis in the limited experimental observations of population and individual behaviour.

Computer models can be classified into two categories according to their method of treating subject occupants [9]. The first category describes building occupants from global perspective, treating the occupants as homogenous ensemble and ignoring individualities in the population. The second category describes building occupants from individual perspective. The first category methods for modelling the occupant evacuation process, by and large, adopt deterministic approaches. They have been criticised for not being able to integrate many human factors and for adopting the assumption that all occupants in a building have similar (if not the same) characteristics and will reach a place of safety within the calculated time [10]. The second category, on the other hand, has stringent demand on the data regarding the vast variety of individual identities.

The methodology discussed in this paper incorporates a non-deterministic, or stochastic, modelling approach into the global descriptions of building occupants beheviour and the effect on the evacuation process. 


\subsection{Treating Egress as a Stochastic Process}

During an emergency evacuation of a building, every individual reacts differently to the cues that are available to the occupants. Every individual also adjusts his or her behaviour according to the surrounding environment, their knowledge of the building and their understanding of the threat. It is impossible to model every detailed aspect of human behaviour in deterministic sense. Yet, it is possible to model the ensemble behaviour in probabilistic sense.

There exist uncertainties in the individual behaviour of building occupants. These uncertainties are reflected in the global perspective as in the number of people who have evacuated the building at a given time or in the total time required for clearing a building. In other words, the number of people who have passed through the exits at a given time is a random variable. This randomness was reflected in the fluctuations in the flow of people from exits and in the amount of time it takes to evacuate an occupied building. Pauls [11] presented the results of uncontrolled total evacuation drills in an office building with 1453 occupants. The total evacuation time was reported to range between 6.6 and 9.2 minutes. The prediction of the average evacuation time using a deterministic model was 7.0 minutes. The time variation in the evacuation drill was attributed by Pauls to the unbalanced demand on the available multiple exits. In a case study of controlled selective evacuation drill, Pauls applied a deterministic model to predict a minimum time of 20 minutes to evacuate an office building of 2100 occupants; the actual recorded time was nearly 30 minutes. Pauls went on to adduce the reasons that contributed to the delays in the evacuation. The variation or uncertainty in the evacuation time was also observed in evacuation drills conducted in the occupancy of large retail stores [12]. Indeed, there are many parameters which can cause the variation in the evacuation time. If a fire drill is repeated many times, one can be certain that the outcome of any single drill will not be exactly the same as that of a deterministic model, neither it will be the same as any other drill. There will be reasons or conditions that lead to a particular outcome. There are a large number of parameters that could influence the outcome, so large that the precise outcome of a drill becomes unpredictable. In the opinion of the present authors, the discrepancy between the results of fire drills and that of a deterministic model does not invalidate the model. Rather, it demonstrates the inability of the deterministic model to cope with the variability and uncertainty that associate with the evacuation process. The challenge in front of us is how to handle these uncertainties in risk assessment and in evaluating the performance of the designed fire safety system. A potent technique one can have recourse to is the probabilistic and stochastic analysis.

Traditional deterministic methods provide us with the means to estimate the average population flow rate through the exit of the building to safety in terms of number of person leaving the building per unit time. The number of occupants remaining in the fire affected part of the building at any given time is the difference of the initial total number of occupants and the number of people who have left the fire affected areas of the building. However, the number of people who have evacuated at a given time is a random variable. One needs to establish an appropriate probability distribution function in order to formulate an expression for risk. In the current study, the analysis starts with the time interval between successive evacuees.

Probabilistic approaches dealing with the uncertainties in the pre-movement time have been discussed by MacLennan et al [13]. It was pointed out that the "time to start 
movement", or the pre-movement time is best described by Weibull distribution

$$
w(t)=\alpha v(v t)^{\alpha-1} \exp \left[-(v t)^{\alpha}\right], \quad t \geq 0,
$$

It is plausible to extend this distribution, with a special constraint, to the time interval between the successive evacuees leaving a building. When the value of the parameter $\alpha$ is set to 1 , the Weibull distribution becomes the exponential distribution

$$
f(t)=v e^{-v t}, \quad t \geq 0 .
$$

The parameter $v$ is called the intensity or population flow rate. It has been proven that the corresponding process of counting the number of people leaving the building, $n$, is a Poisson process governed by the Poisson distribution [15]

$$
P(n, v, t)=e^{-v t}(v t)^{n} / n !, t>0 \text { and } n=0,1, \ldots
$$

The Poisson distribution has been successfully used for modelling purposes in numerous practical applications, e.g. the arrival processes for queuing systems or demand processes for inventory systems [14], which are similar to the evacuation processes in buildings.

The population flow rate $v$ through the exits may be a function of time itself. It is plausible to assume that prior to the evacuation or the onset of movement, the population in the building remains steady and hence $v=0$. This assumption is appropriate for many types of occupancies, for example, hotel and residential dwellings during night time, and movie theatre during a show. In a business environment, this assumption means that the number of people entering the building per unit time is equal to the number of people leaving the building. During a fire emergency the population out-flow rate becomes a finite positive value when the building occupants decide to evacuate. The time of the commencement of the occupant movement, $t_{e}$, in the time-line is the zero reference time for the Poisson distribution $P(n, v, \tau)$, where $\tau=t-t_{e}$. For simplicity, the notation $t$ is used in the following text to denote the time from the commencement of occupant movement, unless indicated otherwise.

\subsection{Treating Uncertainty in the Time to Untenable Conditions}

A difficulty in the evaluation of this uncertainty is that there is very little knowledge of the probability distribution functions for many of the factors that contribute to untenability in an enclosure. It is also generally difficult to derive the probability distribution for the occurrence of untenability from the probability distributions of all the relevant parameters. However, it is generally possible with reasonable accuracy and confidence to determine ranges of times during which the events might occur. Then, uniform probability density distributions may be assumed over these ranges [15].

For a particular fire scenario, the average time to untenability $t_{\mathrm{u}}$ and a range of this time $\left(t_{\mathrm{u}}-\Delta t_{\mathrm{u}}, t_{\mathrm{u}}+\Delta t_{\mathrm{u}}\right)$ (refer Figure 1) can be estimated using a deterministic fire model. Along the timeline, the event of untenable condition would occur between $t_{\mathrm{u}}-\Delta t_{\mathrm{u}}$ and $t_{\mathrm{u}}+\Delta t_{\mathrm{u}}$ with the probability density function

$$
f_{u}(t)=\left\{\begin{array}{cl}
\frac{1}{2 \Delta t_{u}}, & \text { for }\left(t_{u}-\Delta t_{u}\right)<t<\left(t_{u}+\Delta t_{u}\right) \\
0, & \text { otherwise }
\end{array}\right.
$$

Note that the shift of zero reference time will not change the form of the above equation. 


\section{ESTIMATE OF NUMBER OF FATALITIES IN A FIRE SCENARIO}

Equipped with the probability distribution function for the given number of occupants who have exited a building at a given time and the probability density distribution function for the occurrence of untenable conditions in a given fire scenario, one is able to calculate the expected number of fatalities that is associated with the scenario. A general expression is discussed first. Then it is combined with the probability and the probability density functions presented earlier to obtain the expression for the special processes.

Let $L$ be the number of initial population in the building, $m$ be the number of population still remaining in the building at time $t$ with the probability $P_{r}(m, t)$. If $n$ is the number of people who have evacuated the building at time $t$, then

$$
m=L-n
$$

Fatalities occur at a given time if the building becomes untenable and some people become trapped. The former event is described by a probability density function $f_{u}(t)$. The probability that the two events happen at a given time is, assuming independence in the occurrences of the events,

$$
P_{r}(m, t) f_{u}(t) d t
$$

The integration of the above expression over the entire time domain is the probability of $m$ people being trapped in the building

$$
\int_{0}^{\infty} P_{r}(m, t) f_{u}(t) d t
$$

The expected fatalities is then expressed as

$$
D=\sum_{m=0}^{L} m \int_{0}^{\infty} P_{r}(m, t) f_{u}(t) d t
$$

The probability that $m$ people still remain in the building at a given time is the same as the probability of $n$ number of people having evacuated the building at that time, $P(n, t)$, i.e.,

$$
P_{r}(m, t)=P(n, t)
$$

Substituting Eqs.(9) and (13) into Eq.(12) yields

$$
D=\sum_{n=0}^{L}(L-n) \int_{0}^{\infty} P(n, t) f_{u}(t) d t
$$

For a uniform distribution of $f_{u}(t)$ as expressed by Eq.(8) and the Poisson distribution for $P(n, t)$ as expressed by Eq.(7), equation (14) can be written as .

$$
D=\frac{1}{2 \Delta t_{u}} \sum_{n=0}^{L}(L-n) \int_{t_{u}-\Delta t_{u}}^{t_{u}+\Delta t_{u}} \frac{(v t)^{n}}{n !} e^{-v t} d t
$$

The integral in the above equation can be expressed in terms of the cumulative Poisson distribution, $P_{c}(n, v, t)$

$$
D=\frac{1}{2 \Delta t \nu} \sum_{n=0}^{L}(L-n)\left[P_{c}(n, \nu, a)-P_{c}(n, v, b)\right]
$$

where $a=t_{u}-\Delta t, b=t_{u}+\Delta t$ and

$$
P_{c}(n, v, t)=e^{-v t} \sum_{i=0}^{n}(v t)^{i} / i ! .
$$


The derivation of Eq.(16) is given in Appendix. A uniform probability density distribution for time to untenable condition and the Poisson distribution for an exact number of people remaining in a building are illustrated in Figure 2. The overlapping of the two functions gives rise to possibility of fatality.

Equation (12) is a general form of the formula for calculating the expected number of fatalities in a given building fire scenario. The forms of probability and probability density distribution functions other than Poisson and uniform distribution functions can be used. For

example, Hasofer and Beck[8] suggested, based on extensive Monte Carlo simulation results, a lognormal distribution for the time to unable condition in a single fire enclosure. When not much is known about the distribution governing the time to untenability except for the range in which the time would vary, the uniform distribution can be used as a first order approximation [16]. The resultant equation [Eq.(16)] for calculating expected number of deaths in given fire scenarios is rather simple and can be easily implemented in a spreadsheet.

\section{PROBABILISTIC RISK ANALYSIS OF COMPLEX SYSTEMS}

The above described method was derived for single scenario cases. It can be easily extended to multiple scenario cases with the aid of event tree analysis. When undertaking risk evaluation for complex systems, event tree analysis can be used to assist in the systematic development and quantification of multiple scenarios [17]. Event trees provide a simple method to represent a wide range of fire scenarios that can occur. During a fire incidence many possible sequences of events may occur, leading to a set of possible specific consequences. Each sequence of events constitutes a specific fire scenario. Suppose that there are $N$ identified fire scenarios in relation to the design of a building fire safety system. Let $j$ denote the $j$ th scenario and $P_{j}$ the probability of its occurrence. If $D_{j}$ denotes the expected number of death in the $j$ th scenario, then the overall expected number of death $E N D$ can be estimated by

$$
E N D=\sum_{j=1}^{N} P_{j} D_{j}
$$

The estimate of the expected number of death for a given fire scenario has been delineated in the previous section. The number of fire scenarios to be considered in the analysis depends on both the complexity of the system in question and the extent of the analysis being undertaken.

Finally, the expected risk to life $(E R L)$ parameter is given as

$$
E R L=E N D \times F_{f} / L
$$

where $F_{f}$ is the frequency of fatal fires. 


\section{APPLICATION}

An application of the above-described method to a risk assessment of multi-storey office building was reported by $\mathrm{He}$ et al [18]. In this assessment, both the deterministic modelling and the probabilistic analysis were employed. The probabilistic analysis of risk to life was carried out on a comparative basis. The risk parameter of a building which was designed in accordance to the design provisions that are deemed to satisfy the performance requirements of the Building Code of Australia 1996 was compared with that of a building design which adopted alternative solutions.

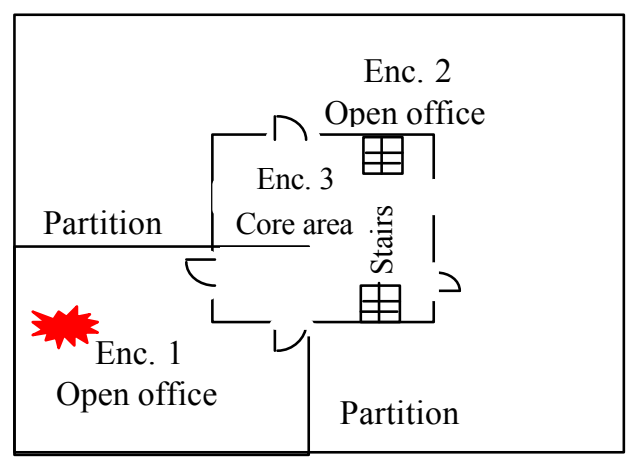

Figure 3. Generic layout of a typical office floor.

A schematic floor layout of the office building in question is given in Figure 3. The floor was divided into three areas, namely, the enclosure of fire origin, that of non-fire origin and the central core area. The building incorporated a range of fire safety measures including sprinklers, fire and smoke barriers, detection and alarm systems, and mechanical smoke control system. A corresponding event tree was established to help identifying possible fire scenarios.

Figure 4 is a portion of the event tree which give rise to 12 scenarios. In this event tree a node to which a symbol " $\mathrm{Y}$ " or " $\mathrm{N}$ " is attached to indicate the status of the corresponding sub-system represents an event. The numbers in the nodes are probabilities of occurrence of the events. The value below a node is the probability of the sequence of the events up to that node. Based on the reliability data and fault tree analysis for various components of the fire safety systems, the probabilities associated with all the identified scenarios, $P_{j}$, were obtained.

The times to untenable condition as the consequence of component failures were determined from deterministic modelling of physical events, using FAST computer model [19] and heat transfer analysis. The times to untenability were evaluated on an enclosure to enclosure basis. The range of time to untenability was determined by selecting a range of the input data (e.g., a range of design fires) for the fire model or heat transfer model. The uniform probability density distribution functions for these times were then determined. Table 1 presents the results of the estimated time range for attainment of untenability in three selected fire scenarios.

The deterministic modelling of occupant movement determined the average time that was required to evacuate the building occupants from fire affected area to a safe place. For the evacuation movement modelling, the deterministic model EVACNET+ [20] was used. Then an estimate of the average population flow rate, $v$, to safety place was obtained by dividing the total number of population by the estimated evacuation time. The EVACNET + model is based on the optimal evacuation calculation scheme and takes into account the effects of travelling distance, travelling speed and possible congestion. 
Sprinkler Fire detection Smoke control Horizontal barrier Beam structure Scenario operation operation operation effective integrity number

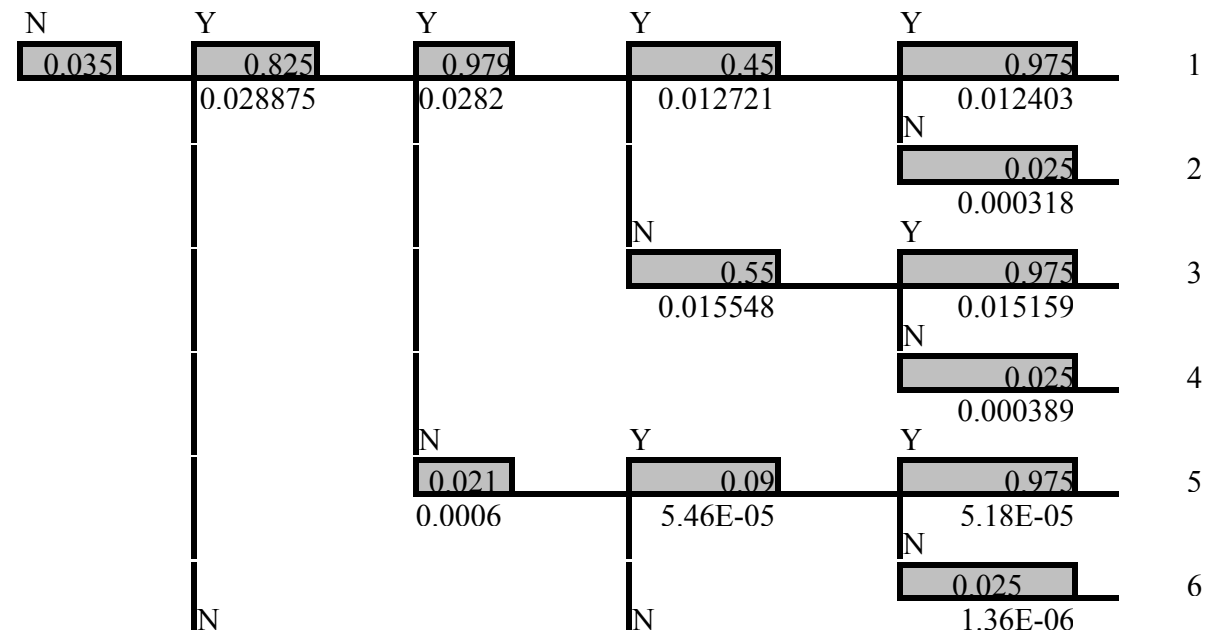

Figure 4. Event tree and probabilities of fire scenarios.

Table 1. Critical times (seconds) and estimated number of fatalities.

\begin{tabular}{|c|c|c|c|c|c|c|c|}
\hline $\begin{array}{r}\text { Scenario } \\
\text { number }\end{array}$ & Location & $t_{\mathrm{e}}$ & $\tau_{\mathrm{u}}{ }^{*}$ & $\Delta t_{\mathrm{u}}$ & $\begin{array}{l}\text { Number of } \\
\text { occupants }\end{array}$ & Fatality & Total \\
\hline \multirow{3}{*}{1} & Enc. 1 & 120 & 40 & 20 & 37 & 0.72 & \multirow{3}{*}{2.91} \\
\hline & Enc. 2 & 230 & 100 & 50 & 113 & 2.19 & \\
\hline & Enc. 3 & 180 & 220 & 110 & 147 & 0 & \\
\hline \multirow[b]{2}{*}{2} & Fire floor & & & & & 2.91 & \multirow[b]{2}{*}{2.945} \\
\hline & Floor above & 360 & 470 & 370 & 150 & 0.035 & \\
\hline \multirow{3}{*}{3} & Enc. 1 & 120 & 30 & 20 & 37 & 1.1 & \multirow{3}{*}{24.0} \\
\hline & Enc. 2 & 230 & 90 & 45 & 113 & 1.3 & \\
\hline & Enc. 3 & 180 & 60 & 30 & 148 & 21.6 & \\
\hline
\end{tabular}

* $\tau_{\mathrm{u}}$ is the time to untenability in the shifted time line, i.e., $\tau_{\mathrm{u}}=t_{\mathrm{u}}-t_{\mathrm{e}}$ and the parameters in Eq.(16) become $a=\tau_{\mathrm{u}}-\Delta t$ and $b=\tau_{\mathrm{u}}+\Delta t$.

The movement commencement time $t_{\mathrm{e}}$ was determined from the pre-movement analysis using the methodologies described by Marchant [21] and was included in Table 1. The initial numbers of occupants in various locations and the calculated expected numbers of fatalities are also included in this table.

The number of fatalities in other given fire scenarios are listed in Table 2 for both the deemed-to-satisfy design (Building A) and the alternative design (Building B). The values as presented in this table were substituted into Eq.(18) to obtain the expected numbers of deaths. The results are presented in Table 3 together with the estimated expected risk to life. 
Table 2. Numbers of deaths and probabilities of various scenarios.

\begin{tabular}{cccc|cccc}
\hline$j$ & $D_{j}$ & $P_{j}(\mathrm{~A})^{*}$ & $P_{j}(\mathrm{~B})$ & $j$ & $D_{j}$ & $P_{j}(\mathrm{~A})$ & $P_{j}(\mathrm{~B})$ \\
\hline 1 & 2.91 & 0.012403 & 0.002362 & 7 & 25.2 & 0.000325 & $6.19 \mathrm{E}-05$ \\
2 & 2.945 & 0.000318 & 0.002362 & 8 & 25.24 & $8.34 \mathrm{E}-06$ & $6.19 \mathrm{E}-05$ \\
3 & 24 & 0.015159 & 0.002887 & 9 & 2.91 & 0.002687 & 0.000512 \\
4 & 24.035 & 0.000389 & 0.002887 & 10 & 2.945 & $6.89 \mathrm{E}-05$ & 0.000512 \\
5 & 2.91 & $5.18 \mathrm{E}-05$ & $1.01 \mathrm{E}-05$ & 11 & 25.2 & 0.0032 & 0.000626 \\
6 & 2.945 & $1.36 \mathrm{E}-06$ & $1.01 \mathrm{E}-05$ & 12 & 25.24 & $8.42 \mathrm{E}-05$ & 0.000626 \\
\hline
\end{tabular}

* $P_{j}(\mathrm{~A})$ is the probability of $j$ th scenario occurring in building $\mathrm{A}$ and $P_{j}(\mathrm{~B})$ is that in building $\mathrm{B}$.

Table 3. Results of evaluation.

\begin{tabular}{ccc}
\hline Parameter & Building A & Building B \\
\hline$E N D$ & 0.7226 & 0.1743 \\
$E R L$ (death per year per population) & $1.63 \times 10^{-5}$ & $3.92 \times 10^{-6}$ \\
\hline
\end{tabular}

The acceptance criterion adopted in the study was that the expected risk to life due to fires in the building with alternative solutions should not be greater than that in the same building if it was designed in accordance with the deemed-to-satisfy provisions of BCA96. In other words, the deemed-to-satisfy building design was used as a reference for the acceptable risk and the following relation must hold

$E R L$ of the alternative system $<E R L$ of the deemed-to-satisfy system

The result of the quantitative analysis demonstrated that the above criterion was indeed satisfied for the particular case studied.

The calculated expected risk to life was also compared with the statistical data available in the literature. For the detailed procedures of quantitative analysis and the numerical results, the reader is referred to [18].

\section{DISCUSSION AND CONCLUSION}

Stochastic approaches dealing with the uncertainties in building fire conditions and the occupant egress processes have been discussed in this paper. General expressions for calculating expected number of deaths in single fire scenario cases were developed. These expressions are believed to be appropriate for the situations where large number of occupants are involved. With the aid of event tree analysis the expression was then extended to complex fire safety systems with multiple fire scenarios. The method can be used to assess and, hence, provide guidance for fire safety engineering design.

The advantage of the method lies in its simplicity. One can make use of the deterministic methods to obtain estimates of a few key parameters, such as the population flow rate through exits and the time range in which untenable conditions may occur. The uncertainties associated with time to untenability and number of occupants trapped in a subject building are then taken into account by applying the postulated Poisson distribution and uniform probability density distribution functions.

The key parameter in the Poisson distribution is the population flow rate. Its value is determined by detection, warning, signage and egress in a building, and by the 
characteristics of the occupants. On the other hand, the fire safety measures, such as the fire protection and suppression systems, in the building will influence the time range of the attainment of untenable conditions, which in turn, will determine the probability density distribution function.

Part of the current work focused on stochastic modelling of the occupant egress process without going into the detailed analysis of individual occupant characteristics based on the behaviour assessment, functional analysis and intervention [22]. To what extent such analysis should be detailed and incorporated into stochastic models still needs further consideration and debate.

\section{REFERENCES}

1 Australian Building Code Board, Fire Safety Engineering Guidelines, 2002 Edition.

2 Meacham, B. J., "Application of Risk Concepts in Performance-Based Fire Protection Engineering", Proceedings of the International Conference on Engineered Fire Protection Design, Society of Fire protection Engineers, 11 - 15 June, 2001, San Francisco, pp.122-131.

3 Ang, A. H-S. and Tang, W. H., Probability Concepts in Engineering Planning and Design, Wiley, New York, 1984.

4 Magnusson, S. E., "Risk Assessment", Proceedings of the Fifth International Symposium on Fire Safety Science, Ed. Hasemi, Y., International Association of Fire Safety Science, Melbourne, 3-7 March, 1997, pp.41-58.

5 Kristiansson, G. H., "On Probabilistic Assessment of Life Safety in Building on fire", Report 5006, Department of Fire Safety Engineering, Lund Institute of Technology, Lund University, 1997.

6 Barry, T. F., "An Introduction to Quantitative Risk Assessment in Chemical Process Industries", The SFPE Handbook of Fire Protection Engineering, 2nd edition, DiNenno, P. J., Editor, the National Fire Protection Association, Boston, 1995, pp.5.102-5.127.

7 Cornel, C. A., "First Order Analysis of Model and Parameter Uncertainty", Proceedings of the International Symposium on Hydrol Water Resources Systems, University of Arizona, Vol. 2, pp.1245-1275, 1972.

8 Hasofer, A. M. and Beck, V. R., "Probability of Death in the Room of Fire Origin: An Engineering Formula", Journal of Fire Protection Engineering, Vol. 10, No. 4, 19-26, 2000.

9 Gwynne, S, Glea, E. R., Owen, M., Lawrence, P. J. and Filippidis, L., "A Review of the Methodologies Used in the Computer Simulation of Evacuation from the Built Environment", Human Behaviour in Fire - Proceedings of the 1st International Symposium, 30 August - 2 September, 1998, Belfast, pp.681-690.

10 Meacham, B. J., "Integrating Human factors Issues into Engineered Fire Safety Design", Human Behaviour in Fire - Proceedings of the First International Symposium, Ed. by T. J. Shields, 31 August - 2 September, 1998, University of Ulster, pp.47-58.

11 Pauls, J., "Movement of People", The SFPE Handbook of Fire Protection Engineering, 2nd edition, DiNenno, P. J., Editor, the National Fire Protection Association, Boston, 1995, pp.3.263-3.285.

12 Shields, T. J. and Boyce, K. E., 2000, "Study of Evacuation From Large Retail Stores", Fire Safety Journal, Vol. 35, No. 1, pp.25-49.

13 MacLennan, H. A., Regan, M. A. and Ware, R., "An engineering Model for the 
Estimation of Occupant Pre-Movement and or Response Times and the Probability of Their Occurrence", Human Behaviour in Fire - Proceedings of the First International Symposium, Ed. by T. J. Shields, 31 August - 2 September, 1998, University of Ulster, pp.13-29.

14 Tijms, H. C., Stochastic Modelling and Analysis: A computational Approach, John Wiley \& Sons, Chichester, 1986, p.18, p.39, p.103.

15 Thomas, I. R., Bennetts, Poon, S. L. and Sims, J. A., 1992, "The Effect of Fire in the Building at 140 William Street", BHP Research, Broken Hill Proprietary Company Limited, Australia.

16 Hall, J. R. Jr., "Probability Concepts", SFPE Handbook, 2nd edition, DiNenno, P. J., Editor, the National Fire Protection Association, Boston, 1995, pp.1.156-1.165.

17 Watts, J. M. Jr., "Fire Risk Ranking", SFPE Handbook, 2nd edition, DiNenno, P. J., Editor, the National Fire Protection Association, Boston, 1995, pp.5.13-5.26.

18 He, Y., Horasan, M., Taylor, P., Ramsay, C. and Lai, D., 2001, "A Probabilistic Fire Safety Engineering Assessment of a Refurbished High Rise Office Building", Proceedings of the International Conference on Engineered Fire Protection Design, Society of Fire protection Engineers, 11 - 15 June, 2001, San Francisco, pp.211-228.

19 Peacock, R. D, Reneke, P. A., Jones, W. W., Bukowski, R. W. and Forney, G. P, "A User's Guide for FAST", National Institute of Standards and Technology, Building and Fire Research Laboratory, USA, 1997.

20 Kisko, T. M. and Francis, R. L., 1985, "EVACNET+: A Computer Program to Determine Optimal Building Evacuation Plans", Fire Safety Journal, 9, pp.211-220.

21 Marchant, R., "Some Discussions on Egress Calculations - Time to Move", International Journal on Engineering Performance-based Fire Code, Vol. 1, No. 2, 1999, pp.81-95.

22 Leslie, J., "Behavioural Safety: Extending the Principles of Applied Behavioural Analysis to Safety in Fires in Public Buildings", Human Behaviour in Fire Proceedings of the First International Symposium, Interscience Communications Ltd, Massachusets Institute of Technology, Cambridge, Boston USA, 26-28 March 2001, pp.1-9.

\section{APPENDIX}

The indefinite counterpart of the definite integral in Eq.(15) can be expressed as

$$
I=\int \frac{(v t)^{n}}{n !} e^{-v t} d t=-\frac{e^{-v t}}{v n !}\left[(v t)^{n}+n(v t)^{n-1}+n(n-1)(v t)^{n-2}+\ldots .+n !\right]=-\frac{e^{-v t}}{v} \sum_{i=0}^{n} \frac{(v t)^{i}}{i !}
$$

The integration constant is, without losing generality, omitted from the above equation. Note that the expression $e^{-v t} \sum_{i=0}^{n}(v t)^{i} / i$ ! is the cumulative Poisson distribution $P_{c}(n, v, t)$. It is the probability that up to $n$ number of counts is registered at a given time $t$. Equation (21) can be written as

$$
I=\int \frac{(v t)^{n}}{n !} e^{-v t} d t=-\frac{1}{v} P_{c}(n, v, t)
$$

Substituting Eq.(22), into Eq.(15) yields Eq.(16). 